OPEN ACCESS

Dipole-Dipole Non-Radiative Energy Transfer Mediated by Surface Plasmons on a Metallic Interface

To cite this article: K. C. Mishra and J. Collins 2019 ECS J. Solid State Sci. Technol. 8 R27

View the article online for updates and enhancements. 


\title{
Dipole-Dipole Non-Radiative Energy Transfer Mediated by Surface Plasmons on a Metallic Interface
}

\author{
K. C. Mishra $\oplus^{1, *}$ and J. Collins $\circledast^{2, * *, z}$ \\ ${ }^{I}$ North Chelmsford, Massachusetts 01863, USA \\ ${ }^{2}$ Department of Physics and Astronomy, Wheaton College, Norton, Massachusetts 02766, USA
}

\begin{abstract}
We have investigated the surface plasmon-mediated energy transfer between two optically active ions in vacuum near a metallic surface using methods of molecular quantum electrodynamics. We have studied the electric dipole-electric dipole energy transfer process only, this being the most dominant mechanism in interionic interaction between two ions in a medium when their wavefunctions do not significantly overlap. The matrix elements for energy transfer, hence energy transfer rates, are calculated using two classes of Feynman diagrams. The intermediate states for one class of diagrams do not satisfy the energy conservation principle, hence they are purely virtual states. Of particular interest are the dependencies of the energy transfer process on (1) the relative positions of the ions with respect to one another projected onto the interface and (2) the distance of each ion from the metal surface. The overall energy transfer process has been found to have both the short range and long-range components, the former being driven by virtual plasmons and the latter by the real plasmons in a non-lossy medium.

(C) The Author(s) 2019. Published by ECS. This is an open access article distributed under the terms of the Creative Commons Attribution 4.0 License (CC BY, http://creativecommons.org/licenses/by/4.0/), which permits unrestricted reuse of the work in any medium, provided the original work is properly cited. [DOI: 10.1149/2.0121902jss]

(cc) BY
\end{abstract}

Manuscript received January 21, 2019. Published February 27, 2019.

Recently there has been considerable interest to utilize surface plasmons in metallic thin films to improve the overall extraction efficiency of luminescent materials ${ }^{1-12}$ and light emitting diodes (LED). ${ }^{13-22}$ The presence of surface plasmons could contribute to providing additional modes to which the emitting ions could radiate thus affecting their radiative lifetime. In a recent publication, Mishra et al. ${ }^{23}$ discussed this aspect of interaction between emitting systems and two-dimensional surface plasmon modes. In the present paper, we address another important process of luminescence, namely, the process of energy transfer ${ }^{24-27}$ between two optical ions in the presence of surface plasmons. It is shown that, similar to the electromagnetic fields associated with radiation, the electromagnetic fields caused by the surface plasmons could facilitate the energy transfer process. Specifically, we have formulated transition rates for nonradiative energy transfer between two ions in vacuum near a metallic thin film by using methods of molecular quantum electrodynamics ${ }^{28}$ to describe a surface plasmonmediated coupling scheme between two optical ions.

Non-radiative energy transfer between two optical ions (Figure 1) is one of the extensively studied phenomena in luminescence. It is also a topic of considerable importance for designing novel phosphor systems. ${ }^{24-26}$ The process can be described as follows: optical ion A is in an excited state due to absorption of a photon. The energy is then transferred from an ion A to a nearby ion, B, resonantly. At the end of the transfer process ion B is found in an excited state and ion A returns to a lower energy state. If this energy transfer process is caused by the emission and subsequent reabsorption of a real photon, it is a radiative transfer process. When the energy transfer is mediated by a virtual photon, a messenger particle that cannot be directly observed, it is known as a non-radiative energy transfer process. In this paper, this resonant, nonradiative energy transfer process is mediated by virtual plasmons associated with the surface plasmon waves at the interface of a metallic layer and a dielectric medium. For simplicity, we have assumed the dielectric medium to be vacuum for developing a quantitative theory of surface plasmon-mediated nonradiative energy transfer. The theory developed here could be easily extended to any dielectric medium in which the optical ions are embedded. However, any broadening of the energy levels, including that which occurs in solids, and its consequence on energy transfer rates have been rigorously treated in the present work.

Since the problem will be formulated in a manner similar to electron-radiation interaction, first we examine the underlying theory of non-radiative energy transfer process via coupling of the radiation

\footnotetext{
*Electrochemical Society Fellow.

**Electrochemical Society Member.

${ }^{z}$ E-mail: jcollins@wheatonma.edu
}

field with the electrons in an optical ion. Dexter ${ }^{29}$ developed the first comprehensive theory of non-radiative energy transfer process in a classic paper in 1953 considering the coulomb interaction between two electrons involved in an energy exchange process as a perturbation driving the process. The interaction Hamiltonian is then obtained in terms of electrostatic multipole-multipole interaction between the two electrons as a function of the interionic distance, $R_{A B}$, by a Taylor series expansion. A first order time-dependent perturbation theory was used to calculate the probability of a transition between an initial composite state described by the ion $\mathrm{A}$ being in the excited state and the ion B in a lower energy (ground) state and a final state in which A is in the ground state and B in the excited state. These composite states describe the ion couple before and after the energy transfer. The perturbing interaction is assumed to be instantaneous. Subsequently, the energy transfer process is described in terms of electric dipole (ED)$\mathrm{ED}, \mathrm{ED}$ - electric quadrupole (QD) and QD-QD interaction between the electrons with similarity to static interaction between two charge distributions.

A more elegant formulation of resonance interaction using ED-ED interaction can be found in Craig and Thirunamachandran ${ }^{28}$ within the framework of molecular quantum electrodynamics. This method can be extended to describe energy transfer processes caused by ED-QD, QD-QD, magnetic dipole (MD)-ED and MD-MD interactions using a higher order approximation of the spatial variation of the vector potential, $\vec{a}$, describing the radiation field near the optical ions. This approach was later adopted to describe ED-MD, ED-ED and MD-MD interactions. ${ }^{30,31}$ In the present manuscript, we extend this formalism to describe an energy transfer process caused by the fields due to surface plasmons.

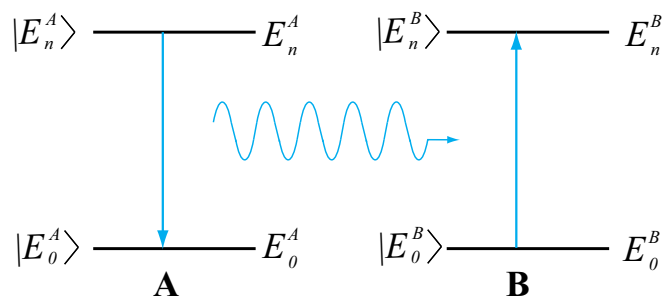

Figure 1. A schematic energy level diagram of nonradiative energy transfer from the donor ion, A to the acceptor ion, B. The energy states and the corresponding energy eigenvalues are indicated. The electronic transitions accompanying the transfer of energy (wavy line) from A to B are indicated by solid arrows. The wavy line in present case corresponds to a virtual plasmon at the interface. 
The adaptation of molecular quantum electrodynamics is possible for any divergence-free electric field associated with a propagating wave. ${ }^{28}$ This is indeed the case for plasmons associated with a propagating surface wave in the interface region between a metal and vacuum. In order to appreciate this assertion one has to understand how the electric multipolar coupling with radiation field occurs. Each ion senses the radiation field through its electric or magnetic multipoles. The energy transfer process between the two ions proceeds by an exchange of virtual photons which by their creation and subsequent annihilation couple the donor decay and acceptor excitation processes over a distance too large for any direct overlap of the wave functions of the electrons. The plasmon-mediated process is similar - the electric field associated with surface plasmons will be sensed by the electric multipoles, and the coupling of the decay and excitation processes occurs through creation of virtual surface plasmons at one site and annihilation at the other. Apart from the fact that the surface plasmons are excited in the plane of the interface, the energy transfer process mediated by plasmons is similar to that by photons.

In order to calculate the transition rate for energy transfer, one needs quantized electromagnetic fields associated with surface plasmons. Fortunately Archambault et al. ${ }^{32}$ have already accomplished this for a lossless medium. These quantized fields have been used in this work to calculate the transition probability for energy transfer from ion A to ion B. Only a case of ED- ED interaction has been studied. An extension to higher order multipoles could be done using a similar approach.

\section{Microscopic Fields Associated with Surface Plasmons}

The fields associated with the surface plasmons in an interface between vacuum and metal has bee extensively studied in the literature. ${ }^{33}$ We describe the field vectors by $\vec{e}, \vec{b}, \vec{d}$ and $\vec{h} . \vec{e}$ and $\vec{b}$ denote electric and magnetic vectors. The auxiliary fields are denoted by $\vec{d}$ and $\vec{h}$. They are given by

$$
\begin{aligned}
\vec{d} & =\varepsilon_{0} \varepsilon \vec{e} \\
\vec{h} & =\mu_{0} \mu \vec{b}
\end{aligned}
$$

$\varepsilon_{0}$ and $\mu_{0}$ represent the electric permittivity and magnetic permeability in the free space, respectively. The dimensionless dielectric constant and relative permeability of a homogeneous medium are denoted by $\varepsilon$ and $\mu$.

In regions free of any charge and current, the Maxwell's equations are given by

$$
\begin{gathered}
\vec{\nabla} \cdot \vec{e}=0 \\
\vec{\nabla} \cdot \vec{b}=0 \\
\vec{\nabla} \times \vec{e}=-\frac{\partial \vec{b}}{\partial t} \\
\vec{\nabla} \times \vec{b}=\varepsilon \mu \varepsilon_{0} \mu_{0} \frac{\partial \vec{e}}{\partial t}
\end{gathered}
$$

For a nonmagnetic medium, Eq. 6 can be expressed as

$$
\vec{\nabla} \times \vec{b}=\varepsilon \varepsilon_{0} \mu_{0} \frac{\partial \vec{e}}{\partial t}=\frac{\varepsilon}{c^{2}} \frac{\partial \vec{e}}{\partial t}=\frac{1}{v^{2}} \frac{\partial \vec{e}}{\partial t}
$$

where $v$ and $c$ represent velocities of the electromagnetic waves in a homogeneous medium and vacuum, respectively, and their dependence on $\varepsilon, \varepsilon_{0}$ and $\mu_{0}$ is implicit in Eq. 7. The electric and magnetic fields can be expressed in terms of the vector potential, $\vec{a}$, and the scalar potential, $\phi$, as

$$
\begin{gathered}
\vec{e}=-\frac{\partial \vec{a}}{\partial t}-\vec{\nabla} \phi \\
\vec{b}=\vec{\nabla} \times \vec{a}
\end{gathered}
$$

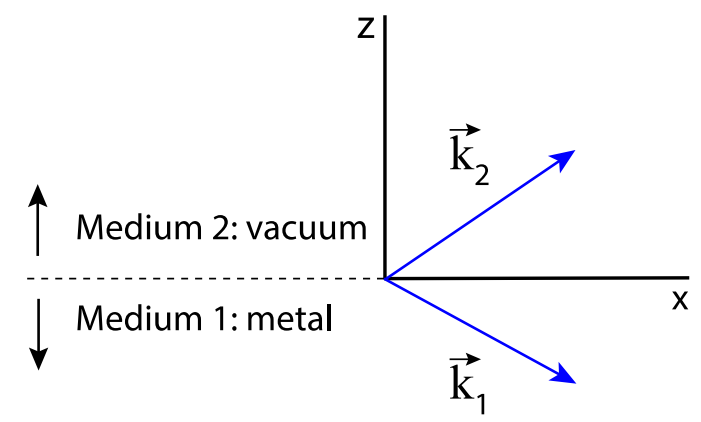

Figure 2. A schematic representation of the interface between metal (medium 1 ) and vacuum (medium 2 ), and the wavevectors $\left(\vec{k}_{1}\right.$ and $\left.\vec{k}_{2}\right)$ of the propagating p-polarized waves in the two media. The choice of the coordinate system used in the text is also indicated.

Since the electric field is divergence free, we choose the scalar potential to vanish everywhere,

$$
\phi=0
$$

Additionally we choose the coulomb gauge in order to quantize the field and use the quantum electrodynamics formalism for energy transfer between two ions discussed earlier,

$$
\vec{\nabla} \cdot \vec{a}=0
$$

The wave equations are then given by

$$
\begin{aligned}
& \nabla^{2} \vec{a}-\frac{1}{v^{2}} \frac{\partial^{2} \vec{a}}{\partial t^{2}}=0 \\
& \nabla^{2} \vec{e}-\frac{1}{v^{2}} \frac{\partial^{2} \vec{e}}{\partial t^{2}}=0 \\
& \nabla^{2} \vec{b}-\frac{1}{v^{2}} \frac{\partial^{2} \vec{b}}{\partial t^{2}}=0
\end{aligned}
$$

The velocity, $v$, in the medium in Eq. 12 to Eq. 14 is given by

$$
v=\left(\frac{1}{\varepsilon_{0} \mu_{0} \varepsilon}\right)^{\frac{1}{2}}
$$

Now we consider the field associated with the surface plasmons on the interface between a metal (medium 1) and vacuum (medium 2). The normal to the interface (Figure 2) is chosen to be the $z$-axis and the interface to be the $x-y$ plane. With respect to an arbitrarily chosen $x$-axis, we define a $p$-polarized wave with wavevectors $\vec{k}_{1}$ and $\vec{k}_{2}$ inside the metallic medium and vacuum respectively. The choice of a $p$-polarized wave is deliberate since this allows solutions to the field equations, Eq. 3 -Eq. 6, that can be considered as a surface wave in the interface. ${ }^{33}$ The fields associated with a $p$-polarized wave can then be described as follows:

For $z \leq 0$ inside metal below the interface,

$$
\begin{gathered}
\vec{h}_{1}=\hat{y} h_{1 y} e^{i\left(k_{1 x} x-k_{1 z} z-\omega t\right)} \\
\vec{e}_{1}=\left(\hat{x} e_{1 x}+\hat{z} e_{1 z}\right) e^{i\left(k_{1 x} x-k_{1 z} z-\omega t\right)}
\end{gathered}
$$

For $z \geq 0$ in the vacuum,

$$
\begin{gathered}
\vec{h}_{2}=\hat{y} h_{2 y} e^{i\left(k_{2 x} x+k_{2 z} z-\omega t\right)} \\
\vec{e}_{2}=\left(\hat{x} e_{2 x}+\hat{z} e_{2 z}\right) e^{i\left(k_{2 x} x+k_{2 z} z-\omega t\right)}
\end{gathered}
$$

The continuity of the tangential component of the electric field at the interface leads to

$$
e_{1 x}=e_{2 x}
$$

$$
k_{1 x}=k_{2 x}=k
$$


We identify $k$ in Eq. 21 as the wavevector of the plasmonic surface wave. Eq. 6 can be rewritten for the auxiliary field, $\vec{h}$, for a medium with dielectric constant $\varepsilon$ as

$$
\vec{\nabla} \times \vec{h}=\varepsilon \varepsilon_{0} \frac{\partial \vec{e}}{\partial t}
$$

In the present case, the dielectric constant of medium 1 is denoted by $\varepsilon$ and that of vacuum as unity. Eq. 22 together with the continuity condition for the tangential component of the electric field in Eq. 20 leads to

$$
\frac{k_{1 z}}{\varepsilon} h_{1 y}+k_{2 z} h_{2 y}=0
$$

The continuity condition for the auxiliary field, $\vec{h}$ at the interface is given by

$$
h_{1 y}=h_{2 y}
$$

A nontrivial solution to Eq. 23 and Eq. 24 leads to the constraint

$$
k_{2 z}=-\frac{k_{1 z}}{\varepsilon}
$$

The wave equation for the auxiliary field, $\vec{h}$ can be shown to satisfy

$$
\nabla^{2} \vec{h}-\varepsilon_{0} \mu_{0} \varepsilon \frac{\partial^{2}}{\partial t^{2}} \vec{h}=0
$$

with the appropriate dielectric constant, $\varepsilon$ for the medium.

Using Eq. 16 for $\vec{h}_{1}$ in medium 1 and Eq. 18 for $\vec{h}_{2}$ in medium 2 in Eq. 26, one obtains

$$
k_{1 z}^{2}+k^{2}=\frac{\varepsilon}{c^{2}} \omega^{2}
$$

and

$$
k_{2 z}^{2}+k^{2}=\frac{\omega^{2}}{c^{2}}
$$

Using $k_{2 z}$ from Eq. 25 in Eq. 27, one can show

$$
k_{2 z}^{2}+\frac{k^{2}}{\varepsilon^{2}}=\frac{1}{\varepsilon} \frac{\omega^{2}}{c^{2}}
$$

On subtracting Eq. 29 from Eq. 28, one obtains the dispersion relations for the surface plasmons,

$$
k^{2}=\frac{\omega^{2}}{c^{2}} \frac{\varepsilon}{1+\varepsilon}
$$

Using Eq. 30, Eq. 27 and Eq. 29, it can be shown that

$$
k_{1 z}^{2}=\frac{\omega^{2}}{c^{2}} \frac{\varepsilon^{2}}{1+\epsilon}
$$

and

$$
k_{2 z}^{2}=\frac{\omega^{2}}{c^{2}} \frac{1}{1+\varepsilon}
$$

When $\omega$ is less than the plasmon frequency, $\omega_{p}, \varepsilon \ll 0$; thus both $k_{1 z}^{2}$ and $k_{2 z}^{2}$ will be imaginary indicating that the fields will attenuate as one moves away from the interface. This is characteristic of a surface wave whose amplitude attenuates with distance from the interface. This allows us to choose two positive attenuation constants, $\alpha_{1}$ and $\alpha_{2}$, that allows the fields to attenuate away from the interface.

Thus,

$$
k_{1 z}=i \alpha_{1}=\sqrt{\frac{\omega^{2}}{c^{2}} \frac{\varepsilon^{2}}{1+\varepsilon}}
$$

Therefore, for $\varepsilon(\omega) \ll 0$, i.e., a frequency regime of interest in this work,

$$
\alpha_{1}=\frac{\omega}{c} \sqrt{\frac{\varepsilon^{2}}{|\varepsilon|-1}}
$$

Similarly, the attenuation constant $\alpha_{2}$ in the vacuum is given by

$$
\alpha_{2}=\frac{\omega}{c} \sqrt{\frac{1}{|\varepsilon|-1}}
$$

The electric fields $\vec{e}_{1}$ and $\vec{e}_{2}$ can now be expressed as

$$
\begin{gathered}
\vec{e}_{1}=\left(\hat{x} e_{1 x}+\hat{z} e_{1 z}\right) e^{\alpha_{1} z} e^{i(k x-\omega t)} \\
\vec{e}_{2}=\left(\hat{x} e_{2 x}+\hat{z} e_{2 z}\right) e^{-\alpha_{2} z} e^{i(k x-\omega t)}
\end{gathered}
$$

Since the electric fields are divergence free (Eq. 3),

$$
e_{1 z}=-\frac{i k}{\alpha_{1}} e_{1 x}
$$

and

$$
e_{2 z}=\frac{i k}{\alpha_{2}} e_{2 x}
$$

Using Eq. 36 and Eq. 38, $\overrightarrow{e_{1}}$ can be expressed as

$$
\vec{e}_{1}=e_{1 x}\left(\hat{x}-\hat{z} \frac{i k}{\alpha_{1}}\right) e^{\alpha_{1} z} e^{i(k x-\omega t)}
$$

Similarly, one obtains from Eq. 37 and Eq. 39

$$
\vec{e}_{2}=e_{2 x}\left(\hat{x}+\hat{z} \frac{i k}{\alpha_{2}}\right) e^{-\alpha_{2} z} e^{i(k x-\omega t)}
$$

Using Eq. 22 and Eq. 33, it can be shown that

$$
\vec{h}_{1}=\hat{y}(-i) \frac{\varepsilon_{0} \epsilon \omega}{\alpha_{1}} e^{\alpha_{1} z} e^{i(k x-w t)}
$$

Similarly, one could express the auxiliary field, $\vec{h}_{2}$, in vacuum to be

$$
\vec{h}_{2}=\hat{y}(-i) \frac{\varepsilon_{0} \varepsilon \omega}{\alpha_{2}} e^{-\alpha_{2} z} e^{i(k x-w t)}
$$

We will focus on the fields in the vacuum $(z>0)$ from here onwards since our interest is to calculate the energy transfer rate between two ions located in this region near a metallic surface. We have also seen that all the field vectors are known if the field amplitude $e_{2 x}$ is known. With an eye to the formulation of this problem using quantum electrodynamics methods, we will first express this field parameter in terms of the vector potential amplitude, $a_{2 x}$ which will be related to plasmon density later. From Eq. 8, we can write

$$
\overrightarrow{e_{2}}=-\frac{\partial \vec{a}_{2}}{\partial t}
$$

From Eq. 41 and Eq. 44, we can assume that

$$
\vec{a}_{2}=a_{2}\left(\hat{x}+\hat{z} \frac{i k}{\alpha_{2}}\right) e^{-\alpha_{2} z} e^{i(k x-\omega t)}
$$

Thus,

$$
\overrightarrow{e_{2}}=i \omega a_{2}\left(\hat{x}+\hat{z} \frac{i k}{\alpha_{2}}\right) e^{-\alpha_{2} z} e^{i(k x-\omega t)}
$$

Since

$$
\vec{b}_{2}=\vec{\nabla} \times \vec{a}_{2}
$$

the magnetic field, $\vec{b}_{2}$ can be expressed as

$$
\vec{b}_{2}=\vec{b} a_{2} e^{-\alpha_{2} z} e^{i(k x-\omega t)}
$$

where

$$
\vec{b}=\left(i k \hat{x}-\alpha_{2} \hat{z}\right) \times\left(\hat{x}+i \frac{k}{\alpha_{2}} \hat{z}\right)
$$




\section{Mode Expansion of the Electromagnetic Fields Associated with the Surface Plasmons}

In order to make a transition to quantum mechanics, the difficulties with normalization of the continuum states are avoided by an analysis of the allowed modes for the electromagnetic fields. ${ }^{28}$ In earlier discussions, we have assumed for convenience that the plasmon wavevector, $\vec{k}$ lies along the $x$-axis. Actually, the plasmon wavevector, $\vec{k}$ is confined to the plane of the interface between metal and vacuum. Therefore, for the purpose of mode counting, we introduce a virtual area of dimension, $s$ with sides of length, $l$ in which the plasmon solutions are confined. The fields are then required to obey periodic boundary conditions, namely, they take the same values on the opposite sides of the square. In this manner, we obtain an infinite but countable discrete set of solutions. The periodic boundary conditions restrict the allowed components of $\vec{k}$ to

$$
k_{x}=\frac{2 \pi n_{x}}{l}, k_{y}=\frac{2 \pi n_{y}}{l}
$$

where $n_{x}$ and $\mathrm{n}_{\mathrm{y}}$ are integers.

Now in the medium 2 , the vector potential can be expressed as

$$
\vec{a}_{2}(\vec{r}, t)=\sum_{\vec{k}}\left(a_{2 \vec{k}}(t) \vec{u}_{2 \vec{k}}(z) e^{i \vec{k} \cdot \vec{\rho}}+\bar{a}_{2 \vec{k}}(t) \overline{\vec{u}}_{2 \vec{k}}(z) e^{-i \vec{k} \cdot \vec{\rho}}\right)
$$

where

$$
\vec{u}_{2 \vec{k}}(z)=\left(\hat{k}+i \frac{k}{\alpha_{2}} \hat{z}\right) \frac{e^{-\alpha_{2} z}}{\sqrt{L(k)}}
$$

and

$$
\vec{r}=\vec{\rho}+z \hat{z}
$$

In Eq. 52 we have introduced another length parameter, $L$, as a function of $\vec{k}$, which allows us to assign a thickness to the surface modes in order to make a transition from a two-dimensional surface to three dimensional volume for proper dimensionality of the density states for use in the Fermi Golden rule. ${ }^{23,32}$ This length parameter, $L$, should not be confused with $l$ introduced here to define the virtual surface area, $s$. Also, in Eq. 53, $\vec{\rho}$ represents a radius vector in the $\mathrm{x}-\mathrm{y}$ plane and $\hat{z}$, the unit vector perpendicular to this plane.

A unit vector along $\vec{k}$ is denoted by $\hat{k}$. Then the electric and magnetic field vectors are given by

$$
\begin{gathered}
\vec{e}_{2}(\vec{r}, t)=i \sum_{\vec{k}} \omega\left(a_{2 \vec{k}}(t) \vec{u}_{2 \vec{k}}(z) e^{i \vec{k} \cdot \vec{\rho}}-\bar{a}_{2 \vec{k}}(t) \overline{\vec{u}}_{2 \vec{k}}(z) e^{-i \vec{k} \cdot \vec{\rho}}\right) \\
\vec{b}_{2}(\vec{r}, t)=i \sum_{\vec{k}}\left(a_{2 \vec{k}}(t) \vec{b}_{2 \vec{k}}(z) e^{i \vec{k} \cdot \vec{\rho}}-\bar{a}_{2 \vec{k}}(t) \overline{\vec{b}}_{2 \vec{k}}(z) e^{-i \vec{k} \cdot \vec{\rho}}\right)
\end{gathered}
$$

\section{Quantization of Plasmonic Fields}

We will follow the scheme for quantization of the plasmonic field originally proposed by Archambault et al. ${ }^{32}$ It was shown that the total energy associated with the surface waves can be expressed as

$$
U=\sum_{\vec{k}} \varepsilon_{0} \omega^{2} s\left(a_{\vec{k}} a_{\vec{k}}^{*}+a_{\vec{k}}^{*} a_{\vec{k}}\right)
$$

One may associate the quantum mechanical annihilation, $\hat{a}_{\vec{k}}$ and creation $\hat{a}_{\vec{k}}^{\dagger}$ operators with the field amplitudes, $a_{\vec{k}}$ and $a_{\vec{k}}^{*}$ in the following manner,

$$
\begin{aligned}
& a_{\vec{k}} \rightarrow\left(\frac{\hbar}{2 \varepsilon_{0} \omega s}\right)^{1 / 2} \hat{a}_{\vec{k}} \\
& a_{\vec{k}}^{*} \rightarrow\left(\frac{\hbar}{2 \varepsilon_{0} \omega S}\right)^{1 / 2} \hat{a}_{\vec{k}}^{\dagger}
\end{aligned}
$$

The Hamiltonian for the surface waves can be expressed as

$$
H_{s p}=\sum_{\vec{k}} \frac{\hbar \omega}{2}\left(\hat{a}_{\vec{k}} \hat{a}_{\vec{k}}^{\dagger}+\hat{a}_{\vec{k}}^{\dagger} \hat{a}_{\vec{k}}\right)
$$

The annihilation and creation operators in Eq. 59 satisfy the following relations,

$$
\begin{gathered}
\hat{a}_{\vec{k}}^{\dagger}\left|n_{\vec{k}}\right\rangle=\sqrt{n_{\vec{k}}+1}\left|n_{\vec{k}}+1\right\rangle \\
\hat{a}_{\vec{k}}\left|n_{\vec{k}}\right|=\sqrt{n_{\vec{k}}}\left|n_{\vec{k}}-1\right\rangle
\end{gathered}
$$

In Eq. 60 and Eq. 61, $n_{\vec{k}}$ is the occupation number for the plasmon mode, $\vec{k}$ and thus an eigenvalue of the (occupation) number operator $\hat{a}_{\vec{k}}^{\dagger} \hat{a}_{\vec{k}}$ corresponding to the eigenvector $\mid n_{\vec{k}}$. Then the operator form of the vector potential and electric and magnetic fields can be expressed as

$$
\begin{aligned}
& \hat{a}_{2}(\vec{r})=\sum_{\vec{k}}\left(\frac{\hbar}{2 \varepsilon_{0} \omega S}\right)^{1 / 2}\left(\hat{a}_{2 \vec{k}} \vec{u}_{2 \vec{k}} e^{i \vec{k} \cdot \vec{\rho}}+\hat{a}_{\vec{k}}^{\dagger} \overline{\vec{u}}_{2 \vec{k}} e^{-i \vec{k} \cdot \vec{\rho}}\right) \\
& \hat{e}_{2}(\vec{r})=i \sum_{\vec{k}}\left(\frac{\hbar \omega}{2 \varepsilon_{0} s}\right)^{1 / 2}\left(\hat{a}_{2 \vec{k}} \vec{u}_{2 \vec{k}} e^{i \vec{k} \cdot \vec{\rho}}-\hat{a}_{\vec{k}}^{\dagger} \overline{\vec{u}}_{2 \vec{k}} e^{-i \vec{k} \cdot \vec{\rho}}\right) \\
& \hat{b}_{2}(\vec{r})=i \sum_{\vec{k}}\left(\frac{\hbar}{2 \varepsilon_{0} \omega s}\right)^{1 / 2}\left(\hat{a}_{2 \vec{k}} \vec{b}_{2 \vec{k}} e^{i \vec{k} \cdot \vec{\rho}}-\hat{a}_{\vec{k}}^{\dagger} \overline{\vec{b}}_{2 \vec{k}} e^{-i \vec{k} \cdot \vec{\rho}}\right)
\end{aligned}
$$

\section{Matrix Element for Energy Transfer Between Two Ions Through Electron-Surface Plasmon Interaction}

Consider two optical ions, A and B located at $\vec{R}_{A}$ and $\vec{R}_{B}$ respectively in vacuum near a metallic surface (region / medium 2 ). The non-relativistic Hamiltonian for a system of these two ions together with the surface plasmon fields on the interface is given by

$$
H=\sum_{\varsigma=A, B} H_{i o n}(\varsigma)+H_{s p}+\sum_{\varsigma=A, B} H_{\text {int }}(\varsigma)
$$

where $H_{\text {ion }}$ corresponds to the Hamiltonian of the system of electrons of the ions, $H_{s p}$ to that of the field associated the surface plasmon waves and $H_{\text {int }}$ to that of interaction between the electrons associated with the ions and the electromagnetic fields associated with the surface wave. The total Hamiltonian, $H$ in Eq. 65 is expressed in a manner similar to that of a system of optical ions and radiation fields in vacuum. ${ }^{28,30,31}$ The exact form of the interaction Hamiltonian is similar to that developed for interaction between radiation fields and ions using a multipolar expansion. This approach is justified since the interaction is mediated by the electromagnetic fields associated with photons or plasmons in the coulomb gauge. Since there are no cross terms involving ions A and B in the interaction Hamiltonian, any interaction between the electrons associated with these ions is mediated by the electromagnetic fields associated with the surface waves travelling at a finite velocity, $v$, given by the plasmon dispersion relation in Eq. 30. Therefore, the interaction potential is fully retarded, not instantaneous, as expected. The first two terms in Eq. 65 refer to the zeroth order Hamiltonian of two canonical systems, one consisting of ions A and $\mathrm{B}$, and the other to that of the surface plasmons derived earlier in Eq. 59, whose eigenstates define the composite states of the ions and the surface plasmons, and the latter in terms of mode occupancy.

The energy transfer process between two ions through interaction with the radiation field within a framework of molecular quantum electrodynamics was discussed earlier. ${ }^{28,30,31}$ The same formalism will be followed here except that the field is caused by the quantized surface waves. The interaction Hamiltonian, $H_{\text {int }}$, is given by

$$
H_{\text {int }}=-\vec{\mu}(A) \cdot \vec{e}_{2}\left(\vec{R}_{A}\right)-\vec{\mu}(B) \cdot \vec{e}_{2}\left(\vec{R}_{B}\right)
$$

where the electric dipole operator for ion $\varsigma$ is given by

$$
\vec{\mu}(\varsigma)=-e \sum_{\alpha}\left(\widehat{\vec{q}}_{\alpha}-\vec{R}_{\varsigma}\right)
$$

In Eq. 67, the summation is over all the electrons, $\alpha$, associated with the ion $\varsigma$. Additionally, $\widehat{\vec{q}}_{\alpha}$ is the position operator of the electron, $\alpha$.

The process of energy transfer from ion A to ion B is schematically described in Fig. 1. The initial state, $|i\rangle$, is described by ion A being in an excited state, $\left|E_{n}^{A}\right\rangle$, corresponding to the energy eigenvalue $E_{n}^{A}$, and ion $\mathrm{B}$ is in a lower energy (ground) state, $\left|E_{0}^{B}\right\rangle$, with energy $E_{0}^{B}$ 


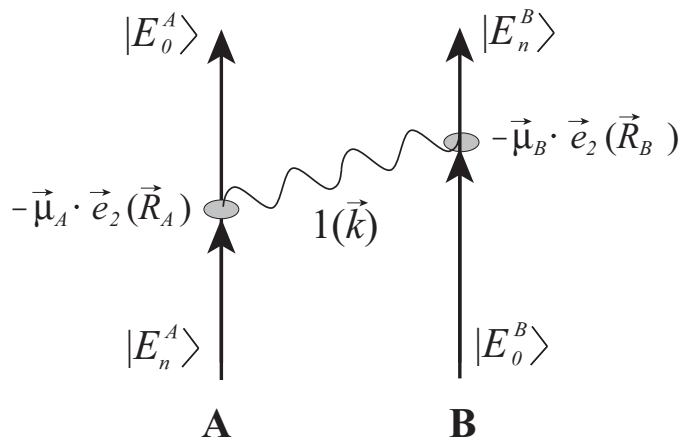

Figure 3. The Feynman diagram corresponding to the intermediate state, $\left|E_{0}^{A}, E_{0}^{B} ; 1(\vec{k})\right\rangle$. The interaction Hamiltonian that drives each ion from the initial to the final state is indicated near the corresponding vortex.

and no plasmons in the interface region. Thus, we express the initial state of the ion-plasmon system as

$$
|i\rangle=\left|E_{n}^{A}, E_{0}^{B} ; 0\right\rangle
$$

with eigenvalue $E_{i}$ given by

$$
E_{i}=E_{n}^{A}+E_{0}^{B}
$$

After the energy is transferred from A to B, A is in a lower (ground) energy state and $\mathrm{B}$ is in an excited state. There are also no plasmons in the interface region. Thus, the final state, $|f\rangle$ is described by the ion A being in the ground state $\left|E_{0}^{A}\right\rangle$ with energy eigenvalue $E_{0}^{A}$ and the ion $\mathrm{B}$ is in the excited state $\left|E_{n}^{B}\right\rangle$ with energy $E_{n}^{B}$ and no plasmons in the interface region. Thus, we express the final state of the ion-plasmon system as

$$
|f\rangle=\left|E_{0}^{A}, E_{n}^{B} ; 0\right\rangle
$$

with eigenvalue, $E_{f}$ given by

$$
E_{f}=E_{0}^{A}+E_{0}^{B}
$$

Since the energy is conserved in the energy transfer process

$$
E_{n o}=E_{n}^{A}-E_{0}^{A}=E_{n}^{B}-E_{0}^{B}
$$

The matrix element for transition from the initial state, $|i\rangle$, to the final state, $|f\rangle$, can be obtained from the second-order perturbation theory to be

$$
M_{f i}=\sum_{I} \frac{\left\langle f\left|H_{\text {int }}\right| I\right\rangle\left\langle I\left|H_{\text {int }}\right| i\right\rangle}{E_{i}-E_{I}}
$$

The summation in Eq. 73 is over intermediate states $|I\rangle$ with energy $E_{I}$. There will be two classes of intermediate states involved in this summation: one with both ions in the ground state and one plasmon with wavevector $\vec{k}$ and the other with both the ions in the excited state and one plasmon with wavevector $\vec{k}$. They lead to two different types of Feynman diagrams (Figures 3 and 4) and their contributions to $M_{f i}$ being $M_{f i}^{1}$ and $M_{f i}^{2}$, respectively. These matrix elements are evaluated separately and summed together to obtain $M_{f i}$.

Before we proceed to evaluate $M_{f i}$ in Eq. 73, we first choose a coordinate system using the position vectors of ions $\mathrm{A}$ and $\mathrm{B}$, and the metal-vacuum interface, the normal to which is chosen as the $\mathrm{z}$-axis. The ion $\mathrm{A}$ is then chosen to be on the $\mathrm{z}$-axis so that its position vector, $\vec{R}_{A}$, is given by

$$
\vec{R}_{A}=Z_{A} \hat{z}
$$

We then choose the position vector of ion $\mathrm{B}$ as

$$
\vec{R}_{B}=X_{B} \hat{x}+Z_{B} \hat{z}
$$

Thus, the interionic vector from $\mathrm{A}$ to $\mathrm{B}, \vec{R}_{A B}$ is given by

$$
\vec{R}_{A B}=X_{B} \hat{x}+\left(Z_{B}-Z_{A}\right) \hat{z}
$$

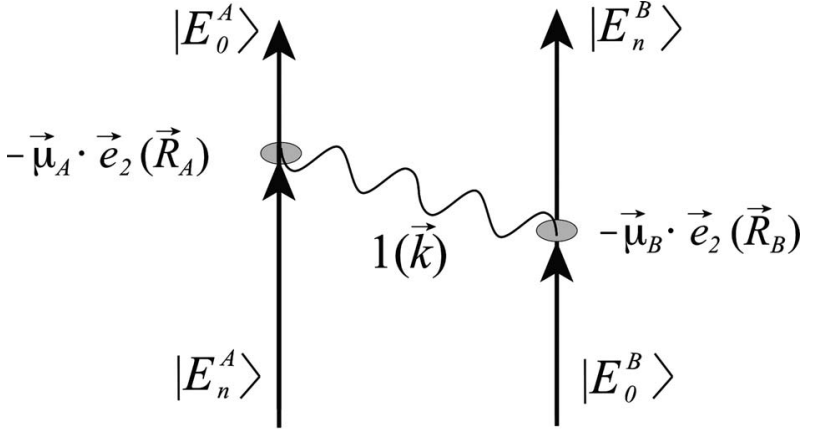

A

B

Figure 4. The Feynman diagram corresponding to the intermediate state, $\left|E_{n}^{A}, E_{n}^{B} ; 1(\vec{k})\right\rangle$. The interaction Hamiltonian that drives each ion from the initial to the final state is indicated near the corresponding vortex. The most interesting feature of this class of intermediate states is that a virtual plasmon is created even when both ions are in the excited sates, thus the energy is not conserved when this intermediate state is created from the initial state.

Therefore, the $x$-axis is defined by the projection of $\vec{R}_{A B}$ on the $x-y$ plane, i.e., the plane of the interface. The $y$-axis is then chosen to be perpendicular to the $x$-z-plane, defined by $\vec{R}_{A B}$, and the $z$-axis to yield a right-handed coordinate system. Any surface wavevector, $\vec{k}$ lies in the $x$ - $y$-plane, i.e.

$$
\vec{k}=\hat{k} k, \hat{k}=\cos \theta \hat{x}+\sin \theta \hat{y}
$$

where $\theta$ denotes the angle between the $x$-axis and the plasmon wavevector, $\vec{k}$. In this coordinate system, $\vec{u}_{2 \vec{k}}$ from Eq. 52 can be expressed as

$$
\vec{u}_{2 \vec{k}}(z)=\left(\cos \theta \hat{x}+\sin \theta \hat{y}+i \frac{k}{\alpha_{2}} \hat{z}\right) \frac{e^{-\alpha_{2} z}}{\sqrt{L(k)}}
$$

Thus, $\overline{\vec{u}}_{2 \vec{k}}$ can now be expressed as

$$
\overline{\vec{u}}_{2 \vec{k}}(z)=\left(\cos \theta \hat{x}+\sin \theta \hat{y}-i \frac{k}{\alpha_{2}} \hat{z}\right) \frac{e^{-\alpha_{2} z}}{\sqrt{L(k)}}
$$

Now we consider contributions from the first class of intermediate states, $|I\rangle$, as shown in Fig. 3,

$$
|I\rangle=\left|E_{0}^{A}, E_{0}^{B} ; 1(\vec{k})\right\rangle
$$

$\vec{k}$ being an arbitrary plasmon wavevector lying in the $x$-y plane. The energy denominator of the matrix element in Eq. 73 is then given by

$$
E_{i}-E_{I}=E_{n o}-\hbar \omega(k)
$$

The dependence of angular frequency, $\omega$ on the plasmon wavevector $\vec{k}$ is determined from the dispersion relation in Eq. 30. Now we can evaluate the numerator of the matrix element $M_{f i}^{1}$ using Eq. 63 for the quantized electric field in region 2 and the states of the composite system in Eq. 68, Eq. 70 and Eq. 80.

Thus,

$$
\left\langle I\left|H_{\text {int }}\right| i\right\rangle=-\left\langle E_{0}^{A}|\vec{\mu}| E_{n}^{A}\right\rangle\left\langle 1(\vec{k})\left|\hat{e}_{2}\left(\vec{R}_{A}\right)\right| 0(\vec{k})\right\rangle
$$

which can be simplified to yield

$$
\begin{aligned}
\left\langle I\left|H_{\text {int }}\right| i\right\rangle= & i\left(\frac{\hbar \omega}{2 \varepsilon_{0} s}\right)^{1 / 2} \frac{e^{-\alpha_{2} Z_{A}}}{\sqrt{L}} \\
& \times\left(\mu_{x}^{0 n}(A) \cos \theta+\mu_{y}^{0 n}(A) \sin \theta-i \frac{k}{\alpha_{2}} \mu_{z}^{0 n}(A)\right) e^{-i k X_{A} \cos \theta}
\end{aligned}
$$


$\mathrm{X}_{\mathrm{A}}$ being the $\mathrm{x}$-coordinate of ion $\mathrm{A}$, which is assumed to be null. Similarly,

$$
\begin{aligned}
\left\langle f\left|H_{\text {int }}\right| I\right\rangle= & -i\left(\frac{\hbar \omega}{2 \varepsilon_{0} s}\right)^{1 / 2} \frac{e^{-\alpha_{2} Z_{B}}}{\sqrt{L}} \\
& \times\left(\mu_{x}^{n 0}(B) \cos \theta+\mu_{y}^{n 0}(B) \sin \theta+i \frac{k}{\alpha_{2}} \mu_{z}^{n 0}(B)\right) e^{i k X_{B} \cos \theta}
\end{aligned}
$$

Using Eq. 81, Eq. 83 and Eq. 84 in Eq. 73, one obtains the contribution to matrix element from this class of intermediate states,

$$
\begin{aligned}
M_{f i}^{1}= & \sum_{\vec{k}}\left(\frac{\hbar \omega}{2 \varepsilon_{0} S}\right) \frac{e^{-\alpha_{2}\left(Z_{A}+Z_{B}\right)}}{L} \frac{e^{i k X_{B A} \cos \theta}}{E_{n 0}-\hbar \omega} \\
& \times\left(\mu_{x}^{0 n}(A) \cos \theta+\mu_{y}^{0 n}(A) \sin \theta-i \frac{k}{\alpha_{2}} \mu_{z}^{0 n}(A)\right) \\
& \times\left(\mu_{x}^{n 0}(B) \cos \theta+\mu_{y}^{n 0}(B) \sin \theta+i \frac{k}{\alpha_{2}} \mu_{z}^{n 0}(B)\right)
\end{aligned}
$$

$\mathrm{X}_{\mathrm{BA}}$ is the separation between ion $\mathrm{B}$ and ion A projected onto the $x$ $y$-plane and is equal to $X_{B}$ in our chosen coordinate system. Now we calculate contributions to the matrix element from the second class of intermediate states, $|I\rangle$, as shown in Fig. 4, given by

$$
|I\rangle=\left|E_{n}^{A}, E_{n}^{B} ; 1(\vec{k})\right\rangle
$$

The corresponding energy denominator is given by

$$
E_{i}-E_{I}=-E_{n o}-\hbar \omega
$$

Proceeding in a manner similar the first case of intermediate states, we obtain

$$
\begin{aligned}
M_{f i}^{2}= & -\sum_{\vec{k}}\left(\frac{\hbar \omega}{2 \varepsilon_{0} s}\right) \frac{e^{-\alpha_{2}\left(Z_{A}+Z_{B}\right)}}{L} \frac{e^{-i k X_{B A} \cos \theta}}{E_{n 0}+\hbar \omega} \\
& \times\left(\mu_{x}^{n 0}(B) \cos \theta+\mu_{y}^{n 0}(B) \sin \theta-i \frac{k}{\alpha_{2}} \mu_{z}^{n 0}(B)\right) \\
& \times\left(\mu_{x}^{0 n}(A) \cos \theta+\mu_{y}^{0 n}(A) \sin \theta+i \frac{k}{\alpha_{2}} \mu_{z}^{0 n}(A)\right)
\end{aligned}
$$

Both the matrix elements, $M_{f i}^{1}$ and $M_{f i}^{2}$ involve summations over the allowed, discrete wavevector modes, $\vec{k}$ in the $x$ - $y$ plane of the interface. The discrete summations over $\vec{k}$ can be replaced by integration in the plane of the interface.

$$
\sum_{\vec{k}} \frac{1}{s}=\int_{0}^{\infty} \int_{0}^{2 \pi} k d k d \theta
$$

First we perform the integration over the polar angle. The only nonzero terms that survive integration over the polar angle involve $1, \cos \theta$, $\sin ^{2} \theta$ and $\cos ^{2} \theta$ only. ${ }^{34}$ Thus we obtain from Eq. 85

$$
\begin{aligned}
M_{f i}^{1}= & \int_{0}^{\infty} \int_{0}^{2 \pi} k d k d \theta\left(\frac{\hbar \omega}{2 \varepsilon_{0}}\right) \frac{e^{-\alpha_{2}\left(Z_{A}+Z_{B}\right)}}{L} \frac{e^{i k X_{B A} \cos \theta}}{E_{n 0}-\hbar \omega}\left(\mu_{x}^{0 n}(A) \mu_{x}^{n 0}(B) \cos ^{2} \theta\right. \\
& +i \frac{k}{\alpha_{2}}\left(\mu_{x}^{0 n}(A) \mu_{z}^{n 0}(B)-\mu_{z}^{0 n}(A) \mu_{x}^{n 0}(B)\right) \cos \theta \\
& \left.+\mu_{y}^{0 n}(A) \mu_{y}^{n 0}(B) \sin ^{2} \theta+\frac{k^{2}}{\alpha_{2}^{2}} \mu_{z}^{0 n}(A) \mu_{z}^{n 0}(B)\right)
\end{aligned}
$$

On integrating over the polar angle, $\theta$, one obtains

$$
\begin{aligned}
M_{f i}^{1}= & \int_{0}^{\infty} k d k\left(\frac{\pi \hbar \omega}{\varepsilon_{0}}\right) \frac{e^{-\alpha_{2}\left(Z_{A}+Z_{B}\right)}}{L} \frac{1}{E_{n 0}-\hbar \omega} \\
& \times\left(\mu_{x}^{0 n}(A) \mu_{x}^{n 0}(B)\left(J_{0}\left(k X_{B A}\right)-\frac{J_{1}\left(k X_{B A}\right)}{k X_{B A}}\right)\right. \\
& +\mu_{y}^{0 n}(A) \mu_{y}^{n 0}(B) \frac{J_{1}\left(k X_{B A}\right)}{k X_{B A}}+\frac{k^{2}}{\alpha_{2}^{2}} \mu_{z}^{0 n}(A) \mu_{z}^{n 0}(B) J_{0}\left(k X_{B A}\right) \\
& \left.-\frac{k}{\alpha_{2}}\left(\mu_{x}^{0 n}(A) \mu_{z}^{n 0}(B)-\mu_{z}^{0 n}(A) \mu_{x}^{n 0}(B)\right) J_{1}\left(k X_{B A}\right)\right)
\end{aligned}
$$

In a similar manner, $M_{f i}^{2}$ can be simplified to

$$
\begin{aligned}
M_{f i}^{2}= & -\int_{0}^{\infty} k d k\left(\frac{\pi \hbar \omega}{\varepsilon_{0}}\right) \frac{e^{-\alpha_{2}\left(Z_{A}+Z_{B}\right)}}{L} \frac{1}{E_{n 0}+\hbar \omega} \\
& \times\left(\mu_{x}^{0 n}(A) \mu_{x}^{n 0}(B)\left(J_{0}\left(k X_{B A}\right)-\frac{J_{1}\left(k X_{B A}\right)}{k X_{B A}}\right)\right. \\
& +\mu_{y}^{0 n}(A) \mu_{y}^{n 0}(B) \frac{J_{1}\left(k X_{B A}\right)}{k X_{B A}}+\frac{k^{2}}{\alpha_{2}^{2}} \mu_{z}^{0 n}(A) \mu_{z}^{n 0}(B) J_{0}\left(k X_{B A}\right) \\
& \left.-\frac{k}{\alpha_{2}}\left(\mu_{x}^{0 n}(A) \mu_{z}^{n 0}(B)-\mu_{z}^{0 n}(A) \mu_{x}^{n 0}(B)\right) J_{1}\left(k X_{B A}\right)\right)
\end{aligned}
$$

In Eq. 91-Eq. 92, $J_{n}$ represent the Bessel functions of first kind of integer order $n$. The quantity, $X_{B A}$ is equal to $X_{B}$, which is the same as the interatomic distance between ions $A$ and $B$ when projected into the $x-y$ plane, and therefore we shall refer to $X_{B A}$ in the future as $\rho$. Now we can obtain the total matrix element $M_{f i}$ from contributions of the two classes of intermediate states listed in Eq. 91 and Eq. 92

$$
M_{f i}=M_{f i}^{1}+M_{f i}^{2}
$$

Thus,

$$
\begin{aligned}
M_{f i}= & \int_{0}^{\infty} k d k\left(\frac{2 \pi \hbar^{2} \omega^{2}}{\varepsilon_{0}}\right) \frac{e^{-\alpha_{2}\left(Z_{A}+Z_{B}\right)}}{L} \frac{1}{E_{n o}^{2}-\hbar^{2} \omega^{2}} \\
& \times\left(\mu_{x}^{0 n}(A) \mu_{x}^{n 0}(B)\left(J_{0}(k \rho)-\frac{J_{1}(k \rho)}{k \rho}\right)\right. \\
& +\mu_{y}^{0 n}(A) \mu_{y}^{n 0}(B) \frac{J_{1}(k \rho)}{k \rho}+\frac{k^{2}}{\alpha_{2}^{2}} \mu_{z}^{0 n}(A) \mu_{z}^{n 0}(B) J_{0}(k \rho) \\
& \left.-\frac{k}{\alpha_{2}}\left(\mu_{x}^{0 n}(A) \mu_{z}^{n 0}(B)-\mu_{z}^{0 n}(A) \mu_{x}^{n 0}(B)\right) J_{1}(k \rho)\right)
\end{aligned}
$$

Since we are considering surface plasmon-mediated energy transfer, it is convenient to express the energy difference between the excited and ground states of the optical ions, $E_{n 0}$, in terms of the plasmon frequencies. Thus, we express

$$
E_{n o}=\hbar \omega(p)=\hbar v(p) p
$$

where the plasmon frequency corresponding to plasmon wavevector, $p$ is denoted by $\omega(p)$, the associated phase velocity being $v(p)$. Unlike photons, the phase velocity of the surface plasmons depends on the plasmon wavenumber, $p$ and is defined by the dispersion relation in Eq. 30, i.e.,

$$
v(p)=c \sqrt{\frac{1+\varepsilon}{\varepsilon}}
$$

Since $\varepsilon$ depends on the plasmon frequency, $\omega$, hence on $p$, the phase velocity is a function of the corresponding plasmon wavenumber, $p$. Because the dominant contribution to the matrix element $M_{f i}$ will come from the pole in Eq. 94 satisfying resonance condition,

$$
E_{n 0}=\hbar \omega
$$


we assume that near resonance, the phase velocity is weakly dependent on the plasmon wavenumber, $k$

$$
v(p)=v(k)=v
$$

Then we can express $M_{f i}$ as

$$
M_{f i}=\int_{0}^{\infty} \frac{f(k)}{k^{2}-p^{2}} d k
$$

where

$$
\begin{aligned}
f(k)= & -\left(\frac{2 \pi}{\varepsilon_{0}}\right) k^{3} \frac{e^{-\alpha_{2}\left(Z_{A}+Z_{B}\right)}}{L}\left(\mu_{x}^{0 n}(A) \mu_{x}^{n 0}(B)\left(J_{0}(k \rho)-\frac{J_{1}(k \rho)}{k \rho}\right)\right. \\
& +\mu_{y}^{0 n}(A) \mu_{y}^{n 0}(B) \frac{J_{1}(k \rho)}{k \rho}+\frac{k^{2}}{\alpha_{2}^{2}} \mu_{z}^{0 n}(A) \mu_{z}^{n 0}(B) J_{0}(k \rho) \\
& \left.-\frac{k}{\alpha_{2}}\left(\mu_{x}^{0 n}(A) \mu_{z}^{n 0}(B)-\mu_{z}^{0 n}(A) \mu_{x}^{n 0}(B)\right) J_{1}(k \rho)\right)
\end{aligned}
$$

The expression for $M_{f i}$ in Eq. 99 is exact except for the assumption about the phase velocity of the surface wave in Eq. 98. Additionally, the attenuation constant $\alpha_{2}$ and the length parameter $L$ in Eq. 100 depend implicitly on the plasmon wavevector through the explicit dependence of $\varepsilon$ on the plasmon frequency. The length parameter, $L$, to be used in the present work is the same as that derived by Archambault et al., ${ }^{32}$ and is given by

$$
L=-\frac{\varepsilon}{2 \alpha_{1}}+\frac{1}{4 \alpha_{2}}\left[\frac{1-\varepsilon}{-\varepsilon} \frac{d(\omega \varepsilon)}{d \omega}-1-\varepsilon\right]
$$

Both $L$ and $\alpha_{2}$ are even functions of $k$ and are real and positive. The integrand in Eq. 99 is an odd function of plasmon wave number, $k$. The integration in Eq. 99 cannot be performed analytically. However, we will assume that most of the contribution will come from the pole near wave number, $p .{ }^{35}$ Evaluating near the pole, $k=p$, we get,

$$
\begin{aligned}
M_{f i} \cong & \left(\frac{\pi}{\varepsilon_{0}}\right) p^{2} \frac{e^{-\alpha_{2}\left(Z_{A}+Z_{B}\right)}}{L}\left(\mu_{x}^{0 n}(A) \mu_{x}^{n 0}(B)\left(J_{0}(p \rho)-\frac{J_{1}(p \rho)}{p \rho}\right)\right. \\
& +\mu_{y}^{0 n}(A) \mu_{y}^{n 0}(B) \frac{J_{1}(p \rho)}{p \rho}+\frac{p^{2}}{\alpha_{2}^{2}} \mu_{z}^{0 n}(A) \mu_{z}^{n 0}(B) J_{0}(p \rho) \\
& \left.-\frac{p}{\alpha_{2}}\left(\mu_{x}^{0 n}(A) \mu_{z}^{n 0}(B)-\mu_{z}^{0 n}(A) \mu_{x}^{n 0}(B)\right) J_{1}(p \rho)\right)
\end{aligned}
$$

\section{Nonradiative Energy Transfer Rate Between Two Optical Ions}

In order to calculate the rate of nonradiative energy transfer rate using the Fermi Golden Rule for randomly oriented electric dipole moments of the optical ions we need to calculate $\left|M_{f i}\right|^{2}$ averaged over all orientations. It can be shown that

$$
\left\langle\mu_{x}^{2}\right\rangle=\left\langle\mu_{y}^{2}\right\rangle=\left\langle\mu_{z}^{2}\right\rangle=\frac{1}{3} \mu^{2}
$$

and

$$
\left\langle\mu_{x} \mu_{y}\right\rangle=\left\langle\mu_{y} \mu_{z}\right\rangle=\left\langle\mu_{z} \mu_{x}\right\rangle=0
$$

where $\mu$ represents the magnitude of the dipole moment of the ion. Using Eq. 102, Eq. 103 and Eq. 104, one can show that

$$
\left\langle\left|M_{f i}\right|^{2}\right\rangle=\frac{\pi^{2}}{9 \varepsilon_{0}^{2}} p^{4}|\mu(A)|^{2}|\mu(B)|^{2} \frac{e^{-2 \alpha_{2}\left(Z_{A}+Z_{B}\right)}}{L^{2}} F(p, \rho)
$$

where $F(p, \rho)$ is given by

$$
\begin{aligned}
F(p \rho)= & J_{0}^{2}(p \rho)\left(1+\frac{p^{4}}{\alpha_{2}^{4}}\right)+\frac{2 J_{1}^{2}(p \rho)}{p^{2} \rho^{2}}\left(1+\frac{p^{4} \rho^{2}}{\alpha_{2}^{2}}\right) \\
& -2 J_{0}(p \rho) \frac{J_{1}(p \rho)}{p \rho}
\end{aligned}
$$

Using Eq. 95 and Eq. 96, it can be shown that

$$
p=\frac{E_{n o}}{\hbar c} \sqrt{\frac{\varepsilon}{1+\varepsilon}}
$$

Substituting $p$ from Eq. 107 in Eq. 105, one obtains

$$
\left\langle\left|M_{f i}\right|^{2}\right\rangle=\frac{\pi^{2}}{9 \varepsilon_{0}^{2}}\left(\frac{E_{n o}}{\hbar c}\right)^{4}\left(\frac{\varepsilon}{1+\varepsilon}\right)^{2}|\mu(A)|^{2}|\mu(B)|^{2} \frac{e^{-2 \alpha_{2}\left(Z_{A}+Z_{B}\right)}}{L^{2}} F(p, \rho)
$$

Using $\alpha_{2}$ from Eq. 35, $F(p, \rho)$ in Eq. 106 can be further simplified to give

$$
\begin{aligned}
F(p \rho)= & J_{0}^{2}(p \rho)\left(1+\frac{p^{4} c^{4}}{\omega^{4}(p)}(|\varepsilon|-1)^{2}\right) \\
& +\frac{2 J_{1}^{2}(p \rho)}{p^{2} \rho^{2}}\left(1+\frac{p^{4} c^{2} \rho^{2}}{\omega^{2}(p)}(|\varepsilon|-1)\right)-2 J_{0}(p \rho) \frac{J_{1}(p \rho)}{p \rho}
\end{aligned}
$$

So far we have not considered the fact that the energy levels of the optical ions are usually broadened for a variety of reasons. The effect of broadening of the energy levels can be described by the line shape function for ion, A for emission, $f_{A}(E)$ and that for ion B by $F_{B}(E)$ for absorption. The line shape functions satisfy the following relations ${ }^{36}$

$$
\begin{aligned}
& \int f_{A}(E) d E=1 ; \int f_{A}(E) E^{n} d E=E_{n o}^{n} \\
& \int F_{B}(E) d E=1 ; \int F_{B}(E) E^{n} d E=E_{n o}^{n}
\end{aligned}
$$

The differential transition rate for energy transfer from ion A to ion B is then given by the Fermi Golden rule,

$$
d \Gamma=\frac{2 \pi}{\hbar}\left\langle\left|M_{f i}\right|^{2}\right\rangle f_{A}(E) F_{B}(E) d E
$$

Using $\left\langle\left|M_{f i}\right|^{2}\right\rangle$ from Eq. 108 in Eq. 112, one obtains

$$
\begin{aligned}
d \Gamma= & \frac{2 \pi^{3}}{9 \varepsilon_{0}^{2} \hbar^{5} c^{4}} E_{n 0}^{4}\left(\frac{\varepsilon(p)}{1+\varepsilon(p)}\right)^{2}|\mu(A)|^{2}|\mu(B)|^{2} \frac{e^{-2 \alpha_{2}\left(Z_{A}+Z_{B}\right)}}{L^{2}} \\
& \times F(p \rho) f_{A}(E) F_{B}(E) d E
\end{aligned}
$$

We can obtain $|\mu(A)|^{2}$ and $|\mu(B)|^{2}$ from the lifetime of ion $A, \tau_{A}$ and integrated absorption intensity of ion $\mathrm{B}, Q(B)$ respectively. They are given by ${ }^{29,31}$

$$
|\mu(A)|^{2}=\frac{3 \pi \varepsilon_{0} c^{3} \hbar^{4}}{E_{n o}^{3}} \frac{1}{\tau_{a}}
$$

and

$$
|\mu(B)|^{2}=\frac{3 \varepsilon_{0} \hbar c}{\pi E_{n 0}} Q(B)
$$

Using $|\mu(A)|^{2}$ and $|\mu(B)|^{2}$ from Eq. 114 and Eq. 115 respectively in Eq. 113, one obtains,

$$
d \Gamma=2 \pi^{3}\left(\frac{\varepsilon(p)}{1+\varepsilon(p)}\right)^{2} \frac{e^{-2 \alpha_{2}\left(Z_{A}+Z_{B}\right)}}{L^{2}} \frac{Q(B)}{\tau_{A}} F(p \rho) f_{A}(E) F_{B}(E) d E
$$

Upon integrating the right-hand side of Eq. 116 over $E$, one obtains the transition rate, $\Gamma$, as

$$
\Gamma=2 \pi^{3}\left(\frac{\varepsilon(p)}{1+\varepsilon(p)}\right)^{2} \frac{e^{-2 \alpha_{2}\left(Z_{A}+Z_{B}\right)}}{L^{2}} \frac{Q(B)}{\tau_{A}} F(p \rho) \int f_{A}(E) F_{B}(E) d E
$$

where we have ignored any explicit dependence of $L$ on energy. 


\section{Results and Discussion}

Eq. 117 gives the nonradiative energy transfer rate, $\Gamma$, between two ions near a metallic surface. Similar to the case of nonradiative energy transfer mediated by photons, $\Gamma$ depends on the overlap of the emission and absorption profiles of the donor and acceptor ions and on the integrated absorption cross-section of the acceptor (ion B) and radiative lifetime of the donor (ion A). ${ }^{29}$ The dependence of $\Gamma$ on the location of the two ions is more interesting. It is governed by two factors. The factor $e^{-2 \alpha_{2}\left(Z_{A}+Z_{B}\right)}$ depends explicitly on the distance of the ion from the plane defined by the metallic surface. The other factor, $F(p \rho)$, depends on the separation of ions A and B projected onto the plane of the interface.

The origin of the first factor is obvious from the dependence of the electric field on the z-coordinates of the ions in Eq. 46. As the ions move away from the interface, they sense a field strength that decreases exponentially. Thus, $\Gamma$ decreases with exponentially with increasing $\mathrm{z}$-coordinate of either ion. The coordinates of the ions are here additive, i.e., it is rather the sum of the $z$-coordinates that determines the exponential decay. This is in contrast to the photon-mediated energy transfer, where the energy transfer rate for a dipole-dipole interaction goes as $R_{A B}^{-6}{ }^{29}$

The second factor, $F(p \rho)$, depends on the separation between the two ions projected onto the $x-y$ plane, $\rho$. This is not surprising since the surface plasmon waves are propagating in this plane. Also, the interface destroys the isotropy of space. $F(p \rho)$ also depends on the dielectric constant of the metal, through the wavevectors $p$ and $\alpha_{2}$ given by Eq. 107 and Eq. 35, respectively. Specifically, $F(p, \rho)$ is a function of the ratio $p / \alpha_{2}$, which has the form

$$
\frac{p}{\alpha_{2}}=\left(\frac{\omega}{c} \sqrt{\frac{\varepsilon}{1+\varepsilon}}\right) \div\left(\frac{\omega}{c} \sqrt{(|\varepsilon|-1)}\right)=\left(\sqrt{\frac{\varepsilon}{1+\varepsilon}}\right) \sqrt{(|\varepsilon|-1)}
$$

For metal in the frequency range of interest, $\varepsilon \leq-1$, so that $1+\varepsilon=$ $-(|\varepsilon|-1)$.

This leads to

$$
\frac{p}{\alpha_{2}}=\sqrt{-\varepsilon}
$$

Thus, for $\varepsilon \leq-1, F(p \rho)$ in Eq. 106 can be written as

$F(p \rho)=J_{0}^{2}(p \rho)\left(1+\varepsilon^{2}\right)+\frac{2 J_{1}^{2}(p \rho)}{p^{2} \rho^{2}}\left(1+\varepsilon p^{2} \rho^{2}\right)-2 J_{0}(p \rho) \frac{J_{1}(p \rho)}{p \rho}$

In order to observe the behavior of this function, let us choose the case of the photon of energy $E_{n 0}=\hbar \omega$, corresponding to $\lambda=500 \mathrm{~nm}$. For a silver interface, the dielectric constant corresponding to $\lambda=500 \mathrm{~nm}$ is $\varepsilon \approx-9 .{ }^{37}$ Under these conditions, $\varepsilon^{2} \approx 81$ so that $F(p \rho)$ becomes

$F(p \rho)=J_{0}^{2}(p \rho)(1+81)+\frac{2 J_{1}^{2}(p \rho)}{p^{2} \rho^{2}}\left(1+9 p^{2} \rho^{2}\right)-2 J_{0}(p \rho) \frac{J_{1}(p \rho)}{p \rho}$

A plot of $F(p \rho)$, normalized to unity at $\rho=0$, is given in Fig. 5. For comparison, also shown in Fig. 5 is the plot of $J_{0}^{2}(p \rho)$, the first term in $F(p \rho)$. Comparing the two plots, it is obvious that the $J_{0}^{2}(p \rho) \epsilon^{2}$ in Eq. 120 is the dominant term, with the other terms playing a minor role. In the visible region, the dielectric constant of silver ranges from $\varepsilon \approx$ -3 at $\lambda=400 \mathrm{~nm}$ to $\varepsilon \approx-22$ at $\lambda=700 \mathrm{~nm}$. Thus, at wavelengths longer than $500 \mathrm{~nm}, \varepsilon$ becomes more negative, and the first term in Eq. 120 ) becomes even more dominant. At shorter wavelengths, $\varepsilon$ becomes less negative, and the first term Eq. 120 becomes less dominant.

For any given wavevector, $p$, the plot of $F(p \rho)$ in Fig. 5 shows that the maximum energy transfer rate occurs when $\rho=0$; that is, when ion $\mathrm{A}$ and ion $\mathrm{B}$ have the same $\mathrm{x}$ and $\mathrm{y}$ coordinates, only differing in their $\mathrm{z}$ coordinates. Thus, the maximum transfer rate occurs when one ion is "on top" of the other as observed from nearest point on the plane of the interface.

As $\rho$ increases, the energy transfer rate initially decreases rapidly. At longer distances, the transfer rate continues to decrease, but much more slowly. Oscillations in $F(p \rho)$ are present at large and small values of $\rho$. The "fast" and "slow" rates of decrease in $F(p \rho)$ indicate the

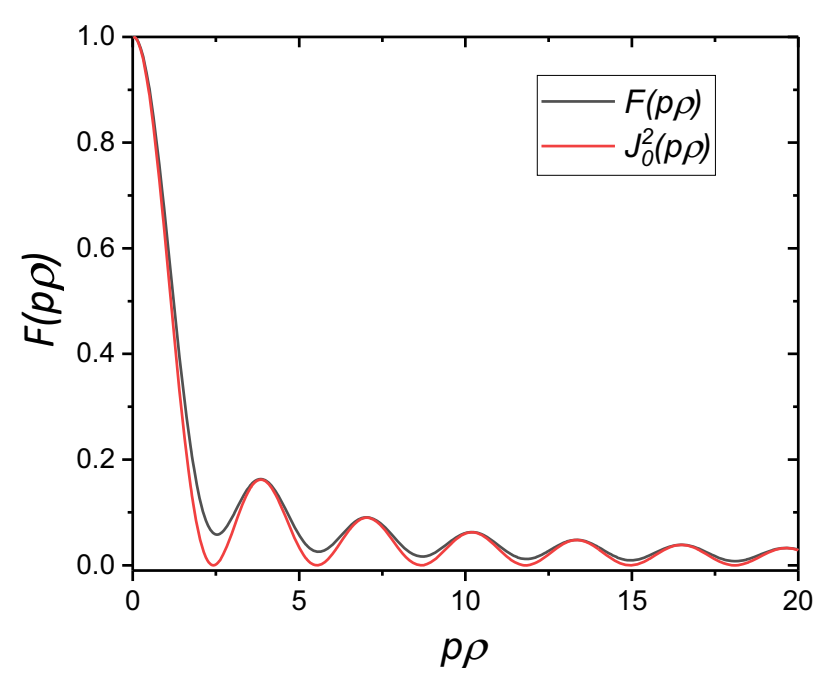

Figure 5. A plot of Eq. 121 (black line) normalized to unity at $\rho=0$. For comparison, also shown is the plot of $J_{0}^{2}(p \rho)$ (red line). Comparing the two plots and examining Eq. 120 and Eq. 121 makes it clear that the $J_{0}^{2}(p \rho) \varepsilon^{2}$ dominates the energy transfer rate. (As in Eq. 121, the plot of $F(p \rho)$ is for a wavelength of $500 \mathrm{~nm}$ and a silver interface.)

presence of both short-range and long-range transfer mechanisms. To estimate the distance of the short-range mechanism, let us assume a surface plasmon of wavelength $500 \mathrm{~nm}$, so $p \sim 2 \pi / 500 \mathrm{~nm}^{-1}$. Using the Fig. 5, we estimate that the "fast" component of the transfer rate extends $p \rho \sim 2.5$, corresponding to $\rho \sim 200 \mathrm{~nm}$, which is less than one wavelength. When $p \rho=50$, however, we find that $\rho \sim 4000$ $\mathrm{nm}$, which is several wavelengths long. Our interpretation is that the short-range mechanism results from energy transfer mediated by primarily by virtual surface plasmons, and the long-range mechanism corresponds formally to energy transfer mediated by primarily by real surface plasmons. Moving from small $\rho$ to large $\rho$, the mediating surface plasmons change from virtual to real in a continuous fashion. An analogous interpretation is also used for the short- and long-range mechanisms in photon-mediated resonance interaction between two molecules. ${ }^{28}$ We note here that we have considered a loss-less medium. In a lossy medium, the long-range interactions will be inhibited.

It is interesting to recall that the formula for $F(p \rho)$ (Eqs. 109 and 120) was derived from Eq. 102, which specifies the role of the various dipole moments responsible for the energy transfer. Table I shows the matrix elements that contribute to each term in Eq. 120) An examination of Eq. 120 and Eq. 121 reveal that the dominant term in $F(p \rho)$ is $J_{0}^{2}(p \rho) \varepsilon^{2}$. As shown in Table I, this term is driven by dipole moments at both ions $\mathrm{A}$ and $\mathrm{B}$ oriented in the z-direction. This observation is consistent with the fact that only a p-polarized wave incident on a metallic interface can produce a surface plasmon. (S-polarized waves have no electric field component normal to the surface, and so cannot establish a surface plasmon.) In plasmon-mediated energy transfer, the main contribution is due to the dipoles oscillating in the $\mathrm{z}$ direction, leading to electric fields preferably oriented normal to the interface, allowing the establishment of surface plasmons, real or virtual. The contributions of the various dipole moments to $F(p \rho)$ evaluated at

Table I. Dipole matrix elements contributions to $F(p \rho)$. Also shown are the percent contribution of the various dipole matrix elements for the case of $\lambda=500 \mathrm{~nm}$ with a silver layer at $\rho=0$.

\begin{tabular}{lcccccccc} 
& $J_{0}^{2}(p \rho)$ & $J_{0}^{2}(p \rho) \varepsilon^{2}$ & $\frac{2 J_{1}^{2}(p \rho)}{p^{2} \rho^{2}}$ & $2 J_{1}^{2}(p \rho) \varepsilon$ & $-2 J_{0}(p \rho) \frac{J_{1}(p \rho)}{p \rho}$ \\
\hline Ion A & $\left|\mu_{x}\right|^{2}$ & $\left|\mu_{z}\right|^{2}$ & $\left|\mu_{x}\right|^{2}$ & $\left|\mu_{y}\right|^{2}$ & $\left|\mu_{z}\right|^{2}$ & $\left|\mu_{x}\right|^{2}$ & $\left|\mu_{x}\right|^{2}$ & $\left|\mu_{y}\right|^{2}$ \\
Ion B & $\left|\mu_{x}\right|^{2}$ & $\left|\mu_{z}\right|^{2}$ & $\left|\mu_{x}\right|^{2}$ & $\left|\mu_{y}\right|^{2}$ & $\left|\mu_{x}\right|^{2}$ & $\left|\mu_{z}\right|^{2}$ & $\left|\mu_{x}\right|^{2}$ & $\left|\mu_{y}\right|^{2}$ \\
$\%$ at & 1.22 & 99.4 & 0.3 & 0.3 & 0 & 0 & -0.61 & -0.61 \\
$\rho=0$ & & & & & & & &
\end{tabular}


$\rho=0$ are also shown in Table I. The $J_{0}^{2}(p \rho) \varepsilon^{2}$ term (when $\varepsilon=-9$ ) is by far the most dominant contributor, accounting for $99.4 \%$ of the energy transfer. Overall, the shape of $F(p \rho)$ follows roughly that of $J_{0}^{2}(p \rho)$, with the maxima and minima occurring roughly at the same values of $p \rho$, and with the main difference that the minima of $F(p \rho)$ do not go to zero.

\section{Conclusions}

We have utilized a molecular quantum electrodynamics approach in multipolar approximation ${ }^{28}$ to the surface plasmon-mediated nonradiative energy transfer between two ions near a metallic interface. The process may be mediated by surface plasmons. The ion-ion energy transfer rate has a distance dependence that reflects the symmetry situation; the transfer rate decreases exponentially with the distances of both ions from the metallic surface, and also decreases as the distance between the two ions projected onto the $x-y$ plane, the plane of the interface. As in the case of photon-mediated energy transfer, the transfer rate depends on the overlap of the emission and absorption profiles of the donor and acceptor, respectively. Though we have assumed the ions to be in vacuum, the theory is easily extended to the case of the ions in a dielectric.

\section{Acknowledgments}

K. C. Mishra wishes to thank Dr. R. Garner and Dr. A. Piquette for many helpful discussions.

\section{ORCID}

K. C. Mishra (D) https://orcid.org/0000-0003-4081-2858

J. Collins (i) https://orcid.org/0000-0002-9567-1039

\section{References}

1. W.-H. Chao, R.-J. Wu, C.-S. Tsai, and T.-B. Wu, J. Appl. Phys., 107, 013101 (2010)

2. S. M. Lee and K. C. Choi, Opt. Express, 18, 12144 (2010).

3. E. Cohen-Hoshen, G. W. Bryant, I. Pinkas, J. Sperling, and I. Bar-Joseph, Nano Lett., 12, 4260 (2012).

4. K. Munechika, Y. Chen, A. F. Tillack, A. P. Kulkarni, I. Jen-La Plante, A. M. Munro, and D. S. Ginger, Nano Lett., 11, 2725 (2011).

5. H. Mertens, J. S. Biteen, H. A. Atwater, and A. Polman, Nano Lett., 6, 2623 (2006)

6. A. Gopinath, S. V. Boriskina, S. Yerci, R. Li, and L. Dal Negro, Appl. Phys, Lett., 96, 071113 (2010).
7. C. Jang, S. M. Lee, and K. C. Choi, Opt. Express, 20, 2143 (2012).

8. H. Mertens and A. Polman, Appl. Phys. Lett., 89, 211107 (2006).

9. L. Xu, Y. Qiang, K. Xiao, Y. Zhang, J. Xie, C. Cui, P. Lin, P. Wang, X. Yu, F. Wu, and D. Yang, Appl. Phys. Lett., 110, 233113 (2017).

10. W. Ye, Q. Huang, X. Jiao, X. Liu, and G. Hu, J. Alloy. Compd., 719, 159 (2017)

11. O. L. Muskens and J. Gómez-Rivas, Mater. Sci. Eng. B, 149, 216 (2008).

12. A. Nikitin, M. Remezani, and J. Gómez-Rivas, ECS J. Solid State Sci. Technol., 5, R3164 (2016)

13. M.-K. Kwon, J.-Y. Kim, B.-H. Kim, I.-K. Park, C.-Y. Cho, C. C. Byeon, and S.-J. Park, Adv. Mater., 20, 1253 (2008)

14. K. Okamoto, I. Niki, A. Shvartser, G. Maltezos, Y. Narukawa, T. Mukai, Y. Kawakami, and A. Scherer, Phys. Stat. Sol. A, 204, 2103 (2007).

15. K. Okamoto, I. Niki, A. Shvartser, Y. Narukawa, T. Mukai, and A. Scherer, Nat Mater, 3, 601 (2004).

16. J. Vučković, M. Lončar, and A. Scherer, IEEE J. Quantum Electron., 36, 1131 (2000)

17. J. Henson, J. DiMaria, E. Dimakis, T. D. Moustakas, and R. Paiella, Opt. Lett., 37, 79 (2012)

18. J. Henson, J. C. Heckel, E. Dimakis, J. Abell, A. Bhattacharyya, G. Chumanov, T. D. Moustakas, and R. Paiella, Appl. Phys. Lett., 95, 151109 (2009).

19. I. Gontijo, M. Boroditsky, E. Yablonovitch, S. Keller, U. K. Mishra, and S. P. DenBaars, Phys. Rev. B, 60, 11564 (1999).

20. N. E. Hecker, R. A. Höpfel, and N. Sawaki, Physica E, 2, 98 (1998).

21. A. Neogi, C.-W Lee, H. O. Everitt, T. Kuroda, A. Tackeuchi, and E. Yablonovitch, Phys. Rev. B, 66, 153305 (2002).

22. G. Lozano, S. R. K. Rodriguez, M. A. Verschuuren, and J. G. Rivas, Light Sci. Appl., 5, e16080 (2016).

23. K. C. Mishra, J. Collins, and A. Piquette, ECS J. Solid State Sci. Technol.,7, R42 (2018).

24. G. Blasse and B. C. Grabmaier, Luminescent Materials, Springer Verlag, New York (1994).

25. Phosphor Handbook, W. M. Yen, S. Shionoya, and H. Yamamoto, Editors, CRC Press, Boca Raton (2007)

26. K. H. Butler, Fluorescent Lamp Phosphors, Pennsylvania State University Press, University Park (1980)

27. B. Di Bartolo, Optical Interaction in Solids, John Wiley \& Sons, New York (1968).

28. D. P. Craig and T. Thirunamachandran, Molecular Quantum Electrodynamics, Dover Publications, New York (1998).

29. D. L. Dexter, J. Chem. Phys., 21, 836 (1953)

30. D. L. Andrews and D. S. Bradshaw, European J. Phys.,25, 845 (2004).

31. K. C. Mishra and J. M. Collins, ECS J. Solid State Sci. Technol.,7, R1 (2018).

32. A. Archambault, F. Marquier, J.-J. Greffet, and C. Arnold, Phys. Rev. B, 82, 035411 (2010).

33. For example see H. Raether, Surface Plasmons on Smooth and Rough Surfaces and on Gratings, Springer-Verlag, Berlin Heidelberg (1988).

34. I. S. Gradshtyen and I. M. Ryzhik, Table of Integrals, Series and Products, Academic, New York (1980)

35. A similar approximation was used by G. W. Ford and W. H. Weber, Phys. Rep., 113, 195 (1984).

36. C. Görller-Walrand and K. Binnemans, Spectral intensities off-ftransitions, in Handbook on the Physics and Chemistry of Rare Earths, (ed. K. A. Gaschneidner Jr. and L. Eyring) Vol. 23, Chapter 167 (1998).

37. H. U. Yang, J. D'Archangel, M. L. Sundheimer, E. Tucker, G. D. Boreman, and M. B. Raschke, Phys. Rev. B, 91, 235137 (2015). 